\title{
Evolutionary Co-Emergence of Appetite and Hormonal Rhythms: A Molecular Highway to Overpass Obesity
}

\section{Akbar Nikkhah}

Chief Highly Distinguished Professor, Department of Animal Sciences, Faculty of Agricultural Sciences, University of Zanjan, Zanjan 313-45195, Iran

*Corresponding author: Akbar Nikkhah, Chief Highly Distinguished Professor, Department of Animal Sciences, Faculty of Agricultural Sciences, University of Zanjan, Zanjan 313-45195, Iran, Tel: +98-24-35052801; Fax: +98-24-35053202; E-mail: anikkha@yahoo.com, nikkhah@znu.ac.ir

Received date: Aug 07, 2014, Accepted date: Aug 11, 2014, Publication date: Aug 31, 2014

Copyright: ( 2014 Nikkhah A. This is an open-access article distributed under the terms of the Creative Commons Attribution License, which permits unrestricted use, distribution, and reproduction in any medium, provided the original author and source are credited.

\section{Editorial}

This perspective article constructs a global and molecular antiadiposity theory on evolutionary circadian rhythms of insulinmediated appetite regulation. Insulin as a storage hormone with leading contributions to intermediary metabolism has been interacting with genetic diversity in different mammals. As demanded by the living environment, insulin stimulates high-performance cellular glucose and amino acids entry [1,2]. Ruminants, as sophisticated working models for studying most devastating diseases in humans, are differently diversified genetically. As such, much less glucose crosses into the portal vein in ruminants than in non-ruminants. Insulin may not have as significant impacts on hepatic glucose metabolism in ruminants versus non-ruminants [1-4].

Nervous system, gut peptides, pancreatic secretions, and nutrient absorption are the main causes of pancreatic insulin release [3]. The nervous system affects insulin release via sympathetic and parasympathetic neurons. The vision, odor, and flavor of foods induce insulin secretion by activating parasympathetic neurons in humans $[4,5]$. The long-time research has led to the proposal that neural impulses and gastrointestinal hormones are involved in postprandial insulin response to food presentation. Secretin and pancreozymin (cholecystokinin) induce insulin release in sheep. Blood insulin in sheep rises sooner than does glucose, suggesting that glucose may not be a major cause of the initial rise in postprandial insulin release. Nevertheless, the ultimate increase in blood glucose may contribute to maintaining the high postprandial insulin levels. Nervous signals (rather than VFA) either directly or through gut hormones may result in post-meal insulin responses. Blood insulin in lactating cows fed once daily during day exhibits distinct circadian rhythms, peaking in the afternoon and falling to a nadir overnight or during the dark phase. A comparable zenith time in blood insulin occurs in the afternoon and early evening.

The evolutionary circadian rhythms of peripheral insulin are closely linked to the rhythms of feed intake. Cows fed forage and concentrate separately exhibit sharp rises in blood insulin upon concentrate deliveries in morning and afternoon and declines in blood insulin shortly thereafter, remaining lower overnight. Similarly, food intake shows two major peaks after concentrate deliveries. Food intake is considerably lower from midnight through dawn, which is similar to humans that are diurnal animals. The fact that insulin works more effectively in morning and early afternoon than in evening and overnight (in human) lends support to the circadian co-rhythmicity of peripheral insulin and food intake [4-7]. These observations and contemplations suggest that circadian rhythms of peripheral blood insulin relate to circadian rhythms of pre- and post-ingestive readily digestible carbohydrates supply. Since human insulin action and peripheral cellular glucose entry are impaired as day leaves for night [4,7-9], prominent nocturnal rhythms of food intake will severely affect insulin efficiency, most likely causing insulin resistance, visceral adiposity, and obesity.

The comparative animal-human genetic diversities acted through molecular mechanisms offer a life-saving perspective to shift the timing of meals from night towards day. This will likely help generate more synchronous rhythms of appetite and insulin. The synchrony will decrease glucose, amino acids, and fatty acids overload overnight when human peripheral cells have limited nutrient assimilation capacity. As a result, adiposity, glucose intolerance, insulin resistance, and metabolic syndrome are less likely to develop. Future research will have to explore chronoendocrinogenomical properties of this molecular action.

\section{Acknowledgments}

Thanks to 'The Ministry of Science, Research and Technology' and 'University of Zanjan' for supporting the author's global programs of optimizing the new millennium science edification.

\section{References}

1. Brockman RP (2005) Glucose and short chain fatty acid metabolism. In Dijkstra, J., J. M. Forbes and J. France. Quantitative aspects of ruminant digestion and metabolism. CABI Publishing. Wallingford 11: 157-176.

2. Brockman RP (1990) Effect of insulin on the utilization of propionate in gluconeogenesis in sheep. Br J Nutr 64: 95-101.

3. Berthoud HR (1984) The relative contribution of the nervous system, hormones, and metabolites to the total insulin response during a meal in the rat. Metabolism 33: 18-25.

4. La Fleur SE (2003) Daily rhythms in glucose metabolism: suprachiasmatic nucleus output to peripheral tissue. J Neuroendocrinol 15: 315-322.

5. Nikkhah A (2012) Eating time modulations of physiology and health: life lessons from human and ruminant models. Iran J Basic Med Sci 15: 891-899.

6. Nikkhah A (2011) Ruminant chronophysiological management: an emerging bioscience. Open Access Anim Physiol 3: 9-12.

7. Nikkhah A (2013) Chronophysiology of ruminant feeding behavior and metabolism: an evolutionary review. Biol. Rhythm Res 44: 197-218.

8. Nikkhah A (2014) Eating Timing and Diabetes. Int. J. Diabetol. Vas. Disease Res 2: 101-102.

9. Nikkhah A ( 2014) When to Eat to Beat Obesity and Diabetes? J. Diab. Metabol. In Press. 